\title{
Chemistry journals impact factors in web of science (ISI) for 2018: a comparative and novel study
}

\begin{abstract}
Journals Impact Factors in Web of Science that are indexed in the article database are ranked using the previous two years' citation data. Impact Factors (IF) is best used to compare journals in the same subject category, as the rate of citation vary widely among disciplines. For example, Nature has an Impact Factor of over 40, while American Historical Review has an impact factor of less than 2. But, both journals are ranked first in their subject category. A comparative and novel study is performed to focus on the impact factors of all available chemistry journals in the world. Despite the progress of science and on-line system, such a comprehensive study on a particular and popular discipline has not been performed. This study uncovers numerous important factors that may identify the quality of a chemistry journal although many other factors may be responsible to judge the meritorious significance of a particular paper.
\end{abstract}

Keywords: Impact factors, ISI, thomson reuters, e- journal, chemistry journal papers, IF, JIF, E journal
Volume 3 Issue 3 - 2019

\author{
Mohammed A Abdulla,' Bimal Krishna Banik² \\ 'Denship of Research, Prince Mohammad Bin Fahd University, \\ Saudi Arabia \\ ${ }^{2}$ Deanship of Research Development; Department of \\ Mathematics and Natural Sciences, Saudi Arabia
}

Correspondence: Abdulla Mohamed A, Prince Mohammad Bin Fahd University Affiliation, Al Khobar, Eastern province, Saudi Arabia, Tel +966/38495489, +966138964566

Email mabdull@pmu.edu.sa

Received: November 06, 2019 | Published: December 12, 2019

\section{Problem statement}

This paper has discussed and comprised the impact factors of chemistry journals published by the ISI for the year 2018. This study has identified the quality and rank of chemistry journals $(638$ E-journals). The impact factor services have provided an overall quality of research output. These data will help the researchers to decide the type of journals in which they like to publish their papers. Our current study will also help researchers to identify papers and the contents that are published in journals that have high impact factors.

\section{Objectives:}

This study aims to achieve the following goals:

1. To recognize and compare the chemistry publications in Clarivate analytics Web of Science (ISI).

2. To compare the impact factors for chemistry journals for the year 2018.

3. To verify all chemistry papers that is cited in Clarivate Analytics Web of Science.

4. Effect of using the journal impact factor (JIF) for chemistry journals for the year 2018.

\section{Research questions:}

This study seeks to answer the following questions:

1. What are the numbers of chemistry publications in Clarivate analytics Web of Science (ISI) in 2018?

2. What are the differences of the Impact factors in chemistry journals for the year 2018 ?
3. How do we use the journal impact factor (JIF)?

4. What are the scientific significances of chemistry journals for the year 2018 ?

\section{Introduction}

The impact factor is a measure of the importance of scientific journals within their field of research. It reflects the citation of research of the published research papers in a particular journal. Thus, the journal that has a high impact factor is important to the researchers. The concept of impact factor was created by Eugene Garfield, the founder of the Institute of Scientific Information (ISI). Some institutions (such as the Thomson Reuters Foundation) currently calculate impact factors annually for published scientific journals. These reports provide information and statistical data for scientific journals in the field of social sciences and sciences, including the impact factor for each journal. These data have been included under the knowledge network services on the Thomson Reuters Foundation website, which are previously published on the Citation Index for Science. ${ }^{1}$

The impact factor of a scientific journal in a given year is calculated at the rate of the times that journal has been cited over the past two years. For example If the impact factor of scientific journal rate of (3) citations in 2018 in that journal the research paper must be published between 2016 and 2017, the impact factor is calculated as follows. ${ }^{2}$

$\mathrm{A}=$ Total number of citations received by all published papers in that journal during 2016 and 2017

$B=$ Number of published articles in the journal's during 2016 and 2017

The impact factor would then be: A/B 
The impact factor of journals is calculated when they complete two years. Clearly, some journals may be affected by this method of calculation if these do not publish on a regular basis as the method of calculation is directly related to the period of time and number of issues. The journal citation reports also provide values for impact factors over a five-year period. ${ }^{3}$

\section{Mechanisms of publishing in ISI journals}

Most of the universities around the world are attempting on a range of future challenges in developing scientific research and promoting education. Perhaps, the most important challenge for the faculties in universities is to publish scientific articles in high impact factor journals.

The database of scientific journals (Institute for Scientific Information, ISI) is recognized to identify the best scientific journals from different countries of the world. This information by ISI is based upon the quality of those journals that reflect a specific level of standard. A paper in ISI-indexed journal indicates a high quality of the content compared to a paper that is published in a journal that is not indexed by ISI.

\section{Material and methods}

The study used mathematical and statistical methods in comparing all published chemistry E-Journals in Clarivate analytics Web of Science (ISI) for the year 2018. This study indicated the strengths and weaknesses in the citation index output in the field of chemistry in ISI.

\section{Analysis of chemistry journal citations}

The process of selecting a journal in Thomson Reuter database is unique. All citations from each journal within the (ISI) are indexed. These citation data is helpful to know the impact of other journals that are under evaluation. The importance of explaining and understanding these statements can be emphasized. The use of quantitative citation data to measure the effect is strong when the journals are in the same scientific field. For example, relatively less explored area, such as Agronomy, do not publish many articles or citations, as like popular areas such as Chemistry or Physics. In some areas, articles take a long time to attract good number of citations. Literature and Humanities belong to this class. But in other areas such as Biosciences, Physics, Chemistry, Engineering and Medicine, it is common to rapidly increase citations every year. These facts must be taken into account when one considers citations of papers on various disciplines.

There are a number of indicators that can be relied upon to try to assess the value and quality of scientific journals, including:

1. Impact factor.

2. The number of a citation for published research in the journal since it is started to function.

3. How long it takes for published research to begin to cite it.

4. The average age of research in which research stops to be cited.

One of the gaps found in calculating the impact factor can be that journals can adopt some policies that raise the value of the impact factor without actually raising the scientific level of this journal. In addition, the sum of citations is in general divided by the number of quotable materials in the calculation of the impact factor. Actually, some journals can reduce the number of citable materials in order to increase the impact factor. Therefore, there can be a debate on the material cited. For example, the editorial of the journals may not be considered a cited material when calculating the impact factor. ${ }^{4}$

Clearly, the impact factor of the journals depends on the individual subjects. For example, the percentage of citation in the first two years ranges from 1 to $3 \%$ if the journal specializes in physics and mathematics, while the percentage rises from 5 to $8 \%$ if the journal specializes in biology. The following data show the analysis of chemistry journals in different fields. ${ }^{4}$

\section{Chemistry multidisciplinary ( 72 journal)}

\section{Aggregate impact factor (Table 1)}

The study showed the total number of citations obtained by the issues of 172 journals in Chemistry Multidisciplinary is 817357 during 2016 to 2017 with an impact factor 5.88 which is higher than other journals in chemistry. ${ }^{5-10}$

\section{Chemistry / physical (I 48 journals)}

\section{Aggregate impact factor (Table 2)}

The study showed the total number of citations obtained by the issues of 148 journals in general chemistry and physics are 752428 during 2016 to 2017 with an impact factor of 5.836.

\section{Chemistry lanalytical (84 journals)}

\section{Aggregate impact factor (Table 3)}

The study showed the total number of citations obtained by the issues of 84 journals in Chemistry and Analytical are 187994 during 2016 to 2017 with an impact factor of 3.728 .

\section{Chemistry applied (7I journals)}

\section{Aggregate impact factor (Table 4)}

The study showed the total number of citations obtained by the issue of 71 journals in Chemistry /Analytical is 115380 during 2016 to 2017 with an impact factor of 3.646 .

\section{Chemistry medical (6I journals)}

\section{Aggregate impact factor (Table 5)}

The study showed the total number of citations obtained by the issue of 61 journals in Medical Chemistry is 82272 during 2016 to 2017 with an impact factor of 2.989 .

\section{Chemistry organic (57 journals)}

\section{Aggregate impact factor (Table 6)}

The study showed the total number of citations obtained by the issue of 57 journals in Organic Chemistry is 127548 during 2016 to 2017, with an impact factor of 3.349 .

\section{Chemistry, inorganic \& nuclear (45 journals)}

\section{Aggregate impact factor (Table 7)}

The study showed the total number of citations obtained by the issue of 45 journals in Chemistry/Inorganic \& Nuclear are 72615 during 2016 to 2017 with an impact factor of 2.861 . 
Table I Aggregate impact factor of I72 journals in chemistry multidisciplinary

\begin{tabular}{lllll}
\hline & $2017 \quad=380400$ & & 2017 & $=68644$ \\
Cites in $\mathbf{2 0 1 8}$ to items published in & $2016 \quad=436957$ & Number of items published in & $2016=70373$ & Sum \\
& Sum $=817357$ & & 139017 \\
& Cites to recent items & 817357 & $=5.88$
\end{tabular}

Table 2 Aggregate impact factor of 148 journals in chemistry/physical

\begin{tabular}{|c|c|c|c|c|c|}
\hline \multirow{3}{*}{ Cites in 2018 to items published in } & 2017 & $=372744$ & & 2017 & $=67053$ \\
\hline & 2016 & $=379684$ & Number of items published in & 2016 & $=61873$ \\
\hline & Sum & $=752428$ & & Sum & $=128926$ \\
\hline \multirow{2}{*}{ Calculation } & \multicolumn{2}{|c|}{ Cites to recent items } & 752428 & \multirow{2}{*}{$=5.836$} & \\
\hline & Numb & recent items & 128926 & & \\
\hline
\end{tabular}

Table 3 Aggregate impact factor of 84 journals in chemistry/analytical

\begin{tabular}{|c|c|c|c|c|c|}
\hline \multirow{3}{*}{ Cites in 2018 to items published in } & 2017 & $=90053$ & & 2017 & $=25240$ \\
\hline & 2016 & $=9794 \mathrm{I}$ & Number of items published in & 2016 & $=25187$ \\
\hline & Sum & $=187994$ & & Sum & $=50427$ \\
\hline \multirow{2}{*}{ Calculation } & \multicolumn{2}{|c|}{ Cites to recent items } & 187994 & \multirow{2}{*}{$=3.728$} & \\
\hline & \multicolumn{2}{|c|}{ Number of recent items } & 50427 & & \\
\hline
\end{tabular}

Table 4 Aggregate impact factor of 71 journals in chemistry/applied

\begin{tabular}{|c|c|c|c|c|c|}
\hline \multirow{3}{*}{ Cites in 2018 to items published in } & 2017 & $=55545$ & \multirow{3}{*}{ Number of items published in } & 2017 & $=16273$ \\
\hline & 2016 & $=59835$ & & 2016 & $=15375$ \\
\hline & Sum & $=115380$ & & Sum & $=31648$ \\
\hline \multirow{3}{*}{ Calculation } & \multicolumn{2}{|c|}{ Cites to recent items } & 115380 & \multirow{3}{*}{$=3.646$} & \\
\hline & \multirow{2}{*}{\multicolumn{2}{|c|}{ Number of recent items }} & & & \\
\hline & & & 31648 & & \\
\hline
\end{tabular}

Table 5 Aggregate impact factor of 61 journals in chemistry/medical

\begin{tabular}{|c|c|c|c|c|c|}
\hline \multirow{3}{*}{ Cites in 2018 to items published in } & 2017 & $=36912$ & & 2017 & $=13696$ \\
\hline & 2016 & $=45360$ & Number of items published in & 2016 & $=13829$ \\
\hline & Sum & $=82272$ & & Sum & $=27525$ \\
\hline \multirow[b]{2}{*}{ Calculation } & \multicolumn{2}{|c|}{ Cites to recent items } & 82272 & \multirow{2}{*}{$=2.989$} & \\
\hline & \multicolumn{2}{|c|}{ Number of recent items } & 27525 & & \\
\hline
\end{tabular}

Table 6 Aggregate impact factor of 57 journals in chemistry/organic

\begin{tabular}{|c|c|c|c|c|c|}
\hline \multirow{3}{*}{$\begin{array}{l}\text { Cites in } 2018 \text { to items } \\
\text { published in }\end{array}$} & 2017 & $=61679$ & \multirow{3}{*}{$\begin{array}{l}\text { Number of items } \\
\text { published in }\end{array}$} & 2017 & $=19218$ \\
\hline & 2016 & $=65869$ & & 2016 & $=18872$ \\
\hline & Sum & $=127548$ & & Sum & $=38090$ \\
\hline \multirow{2}{*}{ Calculation } & \multicolumn{2}{|c|}{ Cites to recent items } & 127548 & \multirow{2}{*}{$=3.349$} & \\
\hline & \multicolumn{2}{|c|}{ Number of recent items } & 38090 & & \\
\hline
\end{tabular}


Table 7 Aggregate impact factor of 45 journals in chemistry/inorganic/nuclear

\begin{tabular}{|c|c|c|c|c|c|}
\hline \multirow{3}{*}{ Cites in 2018 to items published in } & 2017 & $=35289$ & & 2017 & $=12638$ \\
\hline & 2016 & $=37326$ & Number of items published in & 2016 & $=12742$ \\
\hline & Sum & $=72615$ & & Sum & $=25380$ \\
\hline \multirow{3}{*}{ Calculation } & \multicolumn{2}{|c|}{ Cites to recent items } & 72615 & \multirow{3}{*}{$=2.86 \mathrm{I}$} & \\
\hline & \multirow{2}{*}{\multicolumn{2}{|c|}{ Number of recent items }} & & & \\
\hline & & & 25380 & & \\
\hline
\end{tabular}

Table 8 Aggregate impact factor of all subjects in chemistry journals published in 2018

\begin{tabular}{llllll}
\hline Category & Edition & E-journal & Total cites & $\begin{array}{l}\text { Medium impact } \\
\text { factor }\end{array}$ & $\begin{array}{l}\text { Aggregate impact } \\
\text { factor }\end{array}$ \\
Chemistry, Multidisciplinary & SCIE & 172 & $3.833,448$ & 2.260 & 5.880 \\
Chemistry/ Physical & SCIE & 148 & $3,540,659$ & 2.492 & 5.836 \\
Chemistry/Analytical & SCIE & 84 & 960,598 & 2.193 & 3.728 \\
Chemistry Applied & SCIE & 71 & 649,898 & 1.649 & 3.646 \\
Chemistry Medical & SCIE & 61 & 527,979 & 2.530 & 2.989 \\
Chemistry Organic & SCIE & 57 & 790,132 & 2.066 & 3.349 \\
Chemistry, Inorganic \&Nuclear & SCIE & 45 & 435,746 & 2.121 & $2.86 \mathrm{I}$ \\
\hline
\end{tabular}

Source: In cites journal citation reports

\section{Results}

Scientific journals in chemistry are considered within the contents of the ISI Web of Knowledge website. The number of scientific journals published in 2018 was 699, distributed in seven topics as shown in the table below: (Figure 1).

From the above table and figure, the total number of journals in the field of chemistry was found to be 699 in 2018, with $24.6 \%$ journal topics in Chemistry multidisciplinary, while $21.17 \%$ in Physical Chemistry, 20.78\% in Analytical Chemistry, $10.15 \%$ in applied Chemistry, $8.72 \%$ in Medical Chemistry, $8.15 \%$ in organic Chemistry and 6.43 in Inorganic/Nuclear Chemistry. The table also showed the varying percentages of citations for each area because the number of journal titles varies in each area. Therefore, it is essential to calculate the citation of each topic compared to the total citation numbers. For example, the percentage of citation in Inorganic/nuclear chemistry is $5.48 \%$ (Figure 2).

From the chart, the journal impact Factor for multidisciplinary Chemistry is "2.26 JIF" compared with "2.492 JIF" for Physical Chemistry, 2.193 for Analytical Chemistry, "1.649 JIF" for applied Chemistry, "2.53 JIF” for Medical Chemistry, "2.066 JIF” for organic Chemistry, and "2.121 JIF" for inorganic chemistry and Nuclear (Figure 3).

From the chart the Aggregate Journal impact Factor for multidisciplinary in Chemistry is "5.88 JIF" compared with "5.836 JIF" for Physical Chemistry, 3.728 for Analytical Chemistry, "3.646 JIF” for applied Chemistry, "2.989 JIF” for Medical Chemistry, "3.349 JIF" for Organic Chemistry, and "2.861 JIF" for Inorganic Chemistry and Nuclear (Figure 4).

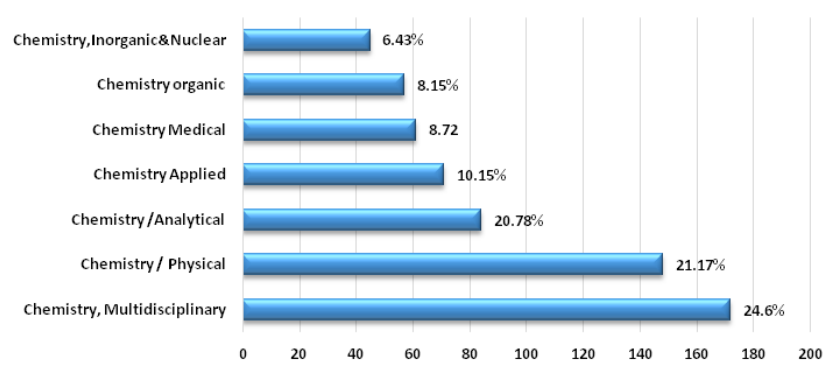

Figure I The chart of the chemistry journals published in 2018.

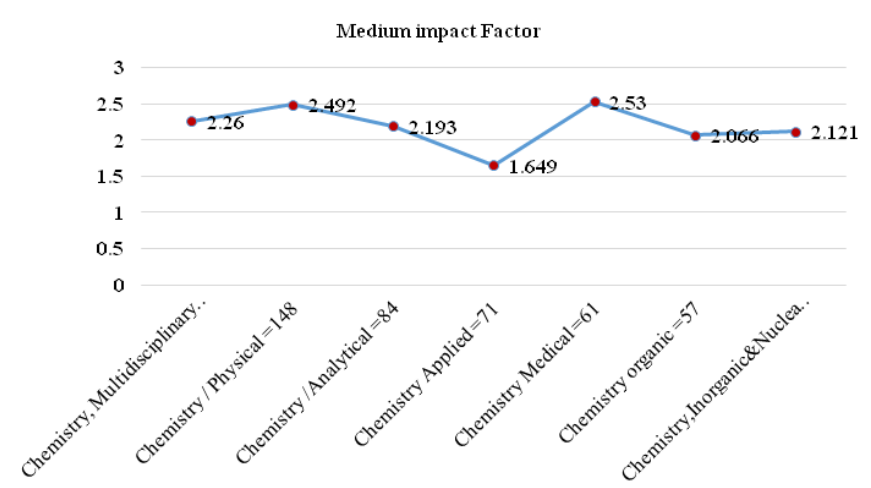

Figure 2 The chart of medium impact factor for the chemistry journals published in 2018. 


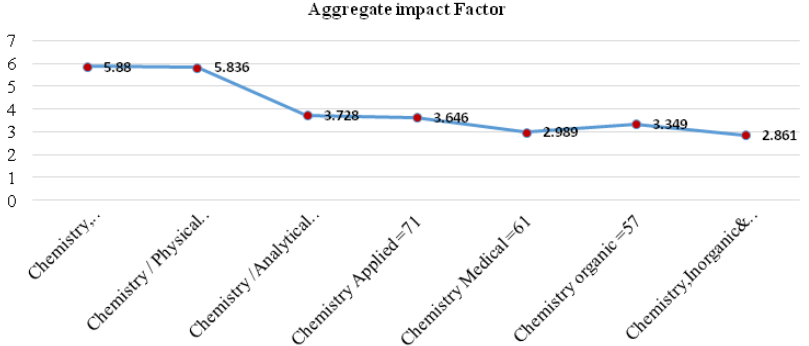

Figure 3 The chart of aggregate impact factor for the chemistry journals published in 2018 .

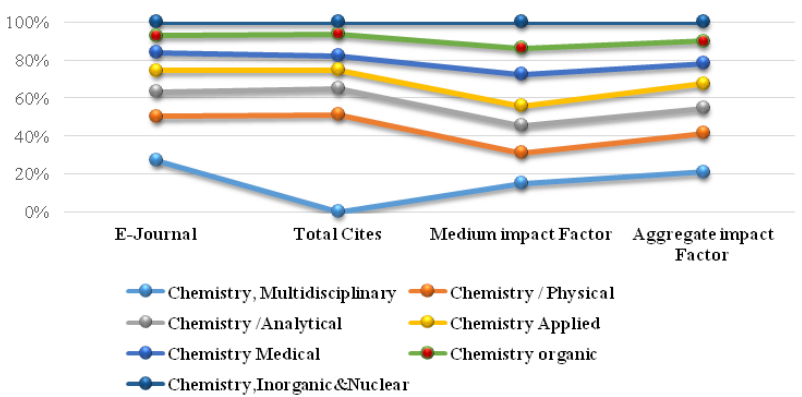

Figure 4 The chart of total citations, medium impact factor and aggregate impact factor of the chemistry journals published in 2018 .

\section{Discussion}

In this article, the authors have compared the impact factors of most of the chemistry journals. A comparative account of the impact factors with respect to a number of situations of diverse chemistry journals is given. This study will help authors to publish papers considering a number of facts and identifying the appropriate journals that suit them. Prior to this study, such an exploratory study was not available. This present work including the general description will be useful to scientists working in the chemistry field as well as other scientific disciplines. This should explore the significance of the results of the work. ${ }^{11-23}$

\section{Conclusion}

This study has identified found the journal impact factor (JIF) effect for Chemistry journals for the year 2018 and found Medical Chemistry has the highest impact factor out of all journals in the various sub-disciplines in chemistry. This subject has 2.53 impact factor followed by Physical Chemistry and Analytical Chemistry. Physical Chemistry has an impact factor of 2.492 and Analytical Chemistry has a value of 1.649. Therefore, Medical Chemistry has the strongest effect on the quality of the research output out of all disciplines in Chemistry.

\section{Acknowledgments}

The authors would like to gratefully acknowledge Library staff, Information Technology at the prince Mohammad Bin Fahd for contributing in this study.

\section{Conflict of interests}

None declared under financial, general, and institutional competing interests.

\section{References}

1. Eugene Garfield. A New Dimension in Documentation through Association of Ideas. Science. 1995;122(3159):108-111.

2. Journal Impact Factor (JIF)

3. Stefan Carlstein. Impact factors: Web of Science. Sweden: Jonkoping University.2016.

4. Introducing the impact factor. Thomson Reuters. 2009.

5. Alberto Martín, Enrique Orduna Malea, Mike Thelwall, et al. Google Scholar, Web of Science, and Scopus: a systematic comparison of citations in 252 subject categories. Journal of Informetrics. 2018;12(4):1160-1177.

6. Aragudige Nagaraja, M Vasanthakumar. Comparison of Web of Science and Scopus Impact Factors of Indian Journals. Library Philosophy and Practice (e-journal). 2011;

7. Clarivate Analytics. Annual Reviews Rankings in Journal Citation Reports (Clarivate Analytics). Impact Factor Rankings. 2019;

8. Erjen van Nierop. Why do statistics journals have low impact factors? Statistica Neerlandica. 2009;63(1):52-62.

9. Gami AS, Montori VM, Wilczynski NL, et al. Author self-citation in the diabetes literature. CMA. 2004;170(13):1925-1927.

10. Garfield E. The Impact Factor and Using It Correctly. Nature. 1997;390(6660):550-550.

11. Groth PT, Gurney T. Studying Scientific Discourse on the Web using Bibliometrics: A Chemistry Blogging Case Study. In: Proceedings of the WebSci10: Extending the Frontiers of Society On-Line Raleigh. NC: Web Science Trust; 2010.

12. Ingwersen P. The calculation of web impact factors. Journal of Documentation. 1998;54(2):236-243.

13. Jo Cross. Impact factors-the basics. The E-Resources Management Handbook, Taylor \& Francis. 2007;

14. Kovacic N, Misak A. Author self-citation in medical literature. CMAJ. 2004;170(13):1929-1930.

15. Kristin Antelman. Do Open-Access Articles Have a Greater Research Impact? USA: Directory of Information Technology at North Carolina State University Libraries; 2004;372-376.

16. Ledley FD, Lovejoy FH. Factors influencing the interests, career paths, and research activities of recent graduates from an academic, pediatric residency program. Houston: US National Library of Medicine; 1993;92(3):436-441.

17. Marashi SA. On the identity of "citers": Are papers promptly recognized by other investigators? Medical Hypotheses. 2005;65(4):822.

18. Mareni Rocha Farias, Bernd Heinrich Storb, Silvia Storpirtis, et al. Impact Factor: an appropriate criterion for the Qualis journals classification in the Pharmacy area? Brazilian Journal of Pharmaceutical Sciences. 2017;53(3).

19. McKiernan EC, Schimanski LA, Munoz Nieves C, et al. Use of the Journal Impact Factor in academic review, promotion, and tenure evaluations. Peer J Preprints. 2019; e27638v2.

20. Nilsson Svenska. The use of impact factors in the research community USA: Curie website; 2016

21. Salah Ahmed Makhloof, Zayd Ahmed Shamsan. Development of specialized journals in Awaqf research in accordance with international publishing standards: Waqf journal as a sample, a research project funded by the Sheikh Rashid Bin Dale Endowed Chair for The Awaqf Studies. Riyadh: The Imam Mohammed Bin Saud University; 2014. 36-44. 
22. Schutte HK, Svec JG. Reaction of Folia Phoniatrica et Logopaedica on the current trend of impact factor measures. Folia phoniatrica et logopaedica. 2007;59(6):281-285.
23. Sherif K Shaheen. UNESCO and the Bibliotheca Alexandrina Regional capacity building workshop on documentary heritage preservation for Arab States. Egypt: Documentary Heritage in Egypt with special reference to Cairo University; 2019. 